\title{
Modeling of Macroeconomics by a Novel Discrete Nonlinear Fractional Dynamical System
}

\author{
Zhenhua Hu and Wen Chen \\ Business School, Central South University, Changsha, Hunan 410083, China \\ Correspondence should be addressed to Wen Chen; chenwen@csu.edu.cn
}

Received 2 July 2013; Accepted 12 November 2013

Academic Editor: Zhen Jin

Copyright ( $2013 \mathrm{Z}$. Hu and W. Chen. This is an open access article distributed under the Creative Commons Attribution License, which permits unrestricted use, distribution, and reproduction in any medium, provided the original work is properly cited.

\begin{abstract}
We propose a new nonlinear economic system with fractional derivative. According to the Jumarie's definition of fractional derivative, we obtain a discrete fractional nonlinear economic system. Three variables, the gross domestic production, inflation, and unemployment rate, are considered by this nonlinear system. Based on the concrete macroeconomic data of USA, the coefficients of this nonlinear system are estimated by the method of least squares. The application of discrete fractional economic model with linear and nonlinear structure is shown to illustrate the efficiency of modeling the macroeconomic data with discrete fractional dynamical system. The empirical study suggests that the nonlinear discrete fractional dynamical system can describe the actual economic data accurately and predict the future behavior more reasonably than the linear dynamic system. The method proposed in this paper can be applied to investigate other macroeconomic variables of more states.
\end{abstract}

\section{Introduction}

Economic dynamics has recently become more prominent in mainstream economics. This influence has been quite pervasive and has influenced both microeconomics and macroeconomics. Its influence in macroeconomics, however, has been much greater. In the real-world life, economic evolution behaves like some process with inner random property. The investigation of economic system gains much development in the recent decades mainly since it can exhibit ubiquitous complex dynamics evidenced by large-amplitude and aperiodic fluctuations [1-3]. For instance, the study of economic system by using van del Pol equation is discussed in [4]. The variation of initial conditions and control parameters of the van del Pol model enables us to understand the periodic, quasiperiodic, and chaotic motion of economic variable considered. In $[5,6]$, the bifurcation topological structure and the global complicated character of a kind of nonlinear financial system are studied. A simplified macroeconomic model discussing the investment, interest rate, and price index is proposed and various evolution results of these economic variables depending on time and parameters are illustrated. Generally speaking, among these prices of literature we may find many mathematical conclusions of economic system, which are helpful to study the dynamical properties of economic models in depth. However, in the above literature, only integer order differential equations are investigated. In the recent years, it is found that depicting the real-world problem by using model with fractional derivative will provide more accurate simulation results.

Fractional calculus, the differentiation and integration with arbitrary order, has been a developing branch of mathematics. It is applied to many scientific and engineering fields successfully in the recent 40 years or so, and it is admitted as a powerful tool in modelling some physical processes with memory effect, which cannot be described well by integer order differential equations. In the current paper, we are not going to discuss the detail of fractional calculus. We refer the readers to [7-14] for more preliminaries. The major difference of fractional derivative is called nonlocal property, which means that the derivative value of the current point depends on all the history function values. This excellent property is suitable for modeling many financial variable series, mainly because of the fact that the financial and economic variable series always exhibit time-dependent memory effect, such as interest rate, stock price, and exchange amount of future [15-18]. Nowadays, although fractional dynamical system has been studied from physical background, there is little work on 
the application of modeling economic variables by fractional derivative, out of which five papers are briefly introduced as follows. In [19], a fractional order financial model based on the fractional Chen system is proposed. It involves the macroeconomic variables such as investment, interest, and price index and exhibits the interesting dynamics behavior of them. In [20], the chaos dynamics and chaos control have been studied using sliding mode method. In [10], the chaos dynamics and chaos control of a class of variable-order fractional financial system have been studied using active control method. The fractional derivative discussed is more general since it depends on the time. In [21], a delayed fractional-order financial system is proposed and the complex dynamical behaviors of this system are discussed by numerical simulations. In [22], a macroeconomic system model with Caputo fractional derivative is proposed firstly as

$$
\begin{aligned}
& D_{t}^{q_{1}} x_{t}=a_{11} x_{t}+a_{12} y_{t}+a_{13} z_{t}+c_{1}, \\
& D_{t}^{q_{2}} y_{t}=a_{21} x_{t}+a_{22} y_{t}+a_{23} z_{t}+c_{2}, \\
& D_{t}^{q_{3}} z_{t}=a_{31} x_{t}+a_{32} y_{t}+a_{33} z_{t}+c_{3}
\end{aligned}
$$

where $x, y$, and $z$ represent the Gross Domestic Product (GDP), inflation, and unemployment, respectively. The subscript $t$ indicates that the variable depends on $t . q_{i} \in(0,1]$, $i=1,2,3$ represents the fractional order of the derivatives, in which the Caputo definition of fractional differentiation is used. The Caputo definition of fractional differentiation is

$$
{ }_{a}^{C} D_{t}^{q} f(t)=\frac{1}{\Gamma(n-q)} \int_{a}^{t} \frac{f^{n}(s)}{(t-s)^{q-n+1}} d s, \quad n-1 \leq q<n,
$$

where $n$ is a positive natural number. The explicit numerical scheme to approximate the $q$ th order fractional derivative is given by

$$
\underset{\left(k-L_{m} / h\right)}{C} D_{t_{k}}^{q} f(t) \approx h^{-q} \sum_{i=0}^{k} c_{i}^{(q)} f\left(t_{k}-i\right)
$$

where $t_{k}=k h, h$ is the time step of discretization, $L_{m}$ is the memory length, and $c_{i}^{(q)}(i=0,1, \ldots)$ are binomial coefficients. Such an approach allows using the tools of the classical control theory for investigation of the state-space trajectories.

Over the last decade, the dynamics of fractional-order financial and economic systems have been investigated via several mathematical methods (see $[19,21]$ and references therein). However, the physical and natural interpretations of those dynamical phenomena obtained in numerical simulations are still not clear. Moreover, there is very little research work on the studying of macroeconomic variables and their evolution via fractional derivative. The main difficulty is that it is really hard to determine what the expression of the economic model is and how to select the optimal value of order of the fractional derivative. To overcome this problem and according to the various definitions of fractional derivative, we propose a class of discrete fractional nonlinear economic model based on the nonlinear fractional economic model with the Jumarie's fractional derivative. The advantage of using this particular derivative is that we can easily get the corresponding discrete equation by removing the limitation operator and there is no need to worry about the influence of nonlocal property of fractional derivative on the accuracy of approximation. The rest of this paper is organized as follows. In Section 2, the mathematical preliminaries are introduced. In Section 3, the nonlinear dynamic econometric model of financial system with fraction-order derivative is proposed, and the parameters of the discrete nonlinear fractional economic model are estimated by the least squares principal. In Section 4, the empirical study of macroeconomic data of USA is shown. Finally, the conclusion is given in Section 5.

\section{Mathematical Preliminaries}

The most common definitions of fractional derivative are the Caputo fractional derivative (see (2)) and the RiemannLiouville fractional derivative as follows. Suppose that $f(x)$ is a continuous function; then its Riemann-Liouville fractional derivative $D^{q} f(x)$ of order $q$ is defined as $[7,8]$ :

$$
\begin{array}{r}
{ }_{a}^{R} D_{t}^{q} f(t)=\frac{1}{\Gamma(n-q)} \frac{d^{n}}{d t^{n}} \int_{a}^{t} \frac{f(s)}{(t-s)^{q-n+1}} d s \\
n-1 \leq q<n,
\end{array}
$$

where $n>0$ is a natural number. The defect of the above definition is that its corresponding discrete approximation expression has infinitely many terms as the step size goes to zero, which is really inconvenient in application. Thus, we consider the following modified fractional derivative, which is firstly proposed by Jumarie [23]:

$$
D_{t}^{q} f(t)=\lim _{h \rightarrow 0} \frac{\Delta^{q} f(t)}{h^{q}},
$$

where $\Delta^{q}$ is fractional difference, which is defined by the expression

$$
\Delta^{q} f(t)=\sum_{k=0}^{+\infty}(-1)^{k}\left(\begin{array}{l}
n \\
k
\end{array}\right) f(t+(q-k) h)
$$

In order to obtain the relation of fractional difference and traditional integer difference, the following generalized Taylor expansion of fractional order is applied:

$$
f(t+h)=\sum_{k=0}^{+\infty} \frac{h^{q k}}{\Gamma(1+q k)} f^{(q k)}(t), \quad 0<q \leq 1,
$$

where $f^{(q k)}(t)$ is the derivative of order $q k$ of $f(x)$. Then (7) can be rewritten as

$$
\begin{aligned}
f^{(q)}(t)= & \Gamma(1+q) \\
& \times\left[\frac{f(t+h)-f(t)}{h^{q}}-\sum_{k=2}^{+\infty} \frac{h^{q k}}{\Gamma(1+q k)} f^{(q k)}(t)\right],
\end{aligned}
$$


where $0<q \leq 1$. Then, letting $h \rightarrow 0$, one can obtain (see [23]):

$$
\begin{array}{r}
D_{t}^{q} f(t)=f^{(q)}(t)=\lim _{h \rightarrow 0} \frac{\Delta^{q} f(t)}{h^{q}}=\Gamma(1+q) \lim _{h \rightarrow 0} \frac{\Delta f(t)}{h^{q}}, \\
0<q \leq 1 .
\end{array}
$$

The expression of fractional derivative (9) is usually called as the Jumarie's definition of fractional derivative. The merit of the definition comparing to the others is that it provides a more simple discrete form of fractional derivative. In what follows, we construct a novel discrete nonlinear fractional dynamic model based on fractional derivative (9).

\section{Model Description}

In model (1), only linear structure is considered. However, many economic systems illustrate nonlinear behavior in the real-world evolution. Now, we consider a class of more general fractional dynamical system as

$$
\begin{aligned}
& D_{t}^{q_{1}} x_{1}(t)=f_{1}\left(x_{1}(t), x_{2}(t), x_{3}(t)\right), \\
& D_{t}^{q_{2}} x_{2}(t)=f_{2}\left(x_{1}(t), x_{2}(t), x_{3}(t)\right), \\
& D_{t}^{q_{3}} x_{3}(t)=f_{3}\left(x_{1}(t), x_{2}(t), x_{3}(t)\right),
\end{aligned}
$$

where the fractional derivatives are defined in (9), and $f_{i}\left(x_{1}(t), x_{2}(t), x_{3}(t)\right),(i=1,2,3)$ are continuously differentiable and describe the dynamic structure of the macroeconomic. As the same with that in (1), $x_{1}$ is the GDP, and $x_{2}$ presents the inflation, and $x_{3}$ depicts the unemployment rate. Using Taylor's theorem for multivariable function, that is,

$$
\begin{aligned}
f_{i}\left(x_{1}, x_{2}, x_{3}\right) \approx & f_{i}(0,0,0)+\frac{\partial f_{i}}{\partial x_{1}} x_{1}+\frac{\partial f_{i}}{\partial x_{2}} x_{2}+\frac{\partial f_{i}}{\partial x_{3}} x_{3} \\
& +\frac{1}{2} \frac{\partial^{2} f_{i}}{\partial x_{1}^{2}} x_{1}^{2}+\frac{1}{2} \frac{\partial^{2} f_{i}}{\partial x_{2}^{2}} x_{2}^{2} \\
& +\frac{1}{2} \frac{\partial^{2} f_{i}}{\partial x_{3}^{2}} x_{3}^{2}+\frac{\partial^{2} f_{i}}{\partial x_{1} \partial x_{2}} x_{1} x_{2} \\
& +\frac{\partial^{2} f_{i}}{\partial x_{2} \partial x_{3}} x_{2} x_{3}+\frac{\partial^{2} f_{i}}{\partial x_{3} \partial x_{1}} x_{1} x_{3}
\end{aligned}
$$

we arrive at

$$
\begin{aligned}
D_{t}^{q_{1}} x_{1}(t)= & c_{1}+a_{11} x_{1}(t)+a_{12} x_{2}(t)+a_{13} x_{3}(t) \\
& +a_{14} x_{1}(t) x_{2}(t)+a_{15} x_{2}(t) x_{3}(t) \\
& +a_{16} x_{3}(t) x_{1}(t)+a_{17} x_{1}^{2}(t) \\
& +a_{18} x_{2}^{2}(t)+a_{19} x_{3}^{2}(t),
\end{aligned}
$$

$$
\begin{aligned}
D_{t}^{q_{2}} x_{2}(t)= & c_{2}+a_{21} x_{1}(t)+a_{22} x_{2}(t)+a_{23} x_{3}(t) \\
& +a_{24} x_{1}(t) x_{2}(t)+a_{25} x_{2}(t) x_{3}(t) \\
& +a_{26} x_{3}(t) x_{1}(t)+a_{27} x_{1}^{2}(t) \\
& +a_{28} x_{2}^{2}(t)+a_{29} x_{3}^{2}(t),
\end{aligned}
$$$$
D_{t}^{q_{3}} x_{3}^{2}(t)=c_{3}+a_{31} x_{1}(t)+a_{32} x_{2}(t)+a_{33} x_{3}(t)
$$$$
+a_{34} x_{1}(t) x_{2}(t)+a_{35} x_{2}(t) x_{3}(t)
$$$$
+a_{36} x_{3}(t) x_{1}(t)+a_{37} x_{1}^{2}(t)
$$$$
+a_{38} x_{2}^{2}(t)+a_{39} x_{3}^{2}(t) \text {. }
$$

Let

$$
\begin{gathered}
f_{1}\left(x_{1}(t), x_{2}(t), x_{3}(t), A_{1}\right) \\
=c_{1}+a_{11} x_{1}(t)+a_{12} x_{2}(t) \\
+a_{13} x_{3}(t)+a_{14} x_{1}(t) x_{2}(t) \\
+a_{15} x_{2}(t) x_{3}(t) \\
+a_{16} x_{3}(t) x_{1}(t)+a_{17} x_{1}^{2}(t) \\
+a_{18} x_{2}^{2}(t)+a_{19} x_{3}^{2}(t), \\
f_{2}\left(x_{1}(t), x_{2}(t), x_{3}(t), A_{2}\right) \\
=c_{2}+a_{21} x_{1}(t)+a_{22} x_{2}(t) \\
+a_{23} x_{3}(t)+a_{24} x_{1}(t) x_{2}(t) \\
+a_{25} x_{2}(t) x_{3}(t)+a_{26} x_{3}(t) x_{1}(t) \\
+a_{27} x_{1}^{2}(t)+a_{28} x_{2}^{2}(t)+a_{29} x_{3}^{2}(t), \\
f_{3}\left(x_{1}(t), x_{2}(t), x_{3}(t), A_{3}\right) \\
=c_{3}+a_{31} x_{1}(t)+a_{32} x_{2}(t)+a_{33} x_{3}(t) \\
+a_{34} x_{1}(t) x_{2}(t)+a_{35} x_{2}(t) x_{3}(t) \\
+a_{36} x_{3}(t) x_{1}(t)+a_{37} x_{1}^{2}(t)+a_{38} x_{2}^{2}(t) \\
+a_{39} x_{3}^{2}(t), \\
A_{i}=\left(c_{i}, a_{i 1}, a_{i 2}, \ldots, a_{i 9}\right), \quad i=1,2,3
\end{gathered}
$$

and then model (10) can be rewritten as

$$
\begin{aligned}
& D_{t}^{q_{1}} x_{1}(t)=f_{1}\left(x_{1}(t), x_{2}(t), x_{3}(t), A_{1}\right), \\
& D_{t}^{q_{2}} x_{2}(t)=f_{2}\left(x_{1}(t), x_{2}(t), x_{3}(t), A_{2}\right), \\
& D_{t}^{q_{3}} x_{3}(t)=f_{3}\left(x_{1}(t), x_{2}(t), x_{3}(t), A_{3}\right) .
\end{aligned}
$$

In model (14), the pair $\left(q_{i}, A_{i}\right)(i=1,2,3)$ is parameters to be estimated. The fractional definition (9) means that 
$D_{t}^{q_{i}} x_{i}\left(t_{n}\right)$ can be replaced by $\left(\left(x_{i}\left(t_{n+1}\right)-x_{i}\left(t_{n}\right)\right) /\right.$ $\left.\left(t_{n+1}-t_{n}\right)^{q_{i}}\right) \Gamma\left(1+q_{i}\right)$ when $t_{n+1}-t_{n}$ is small enough. Hence we obtain the discrete form of model (14) as follows:

$$
\begin{aligned}
& \frac{x_{1}\left(t_{n+1}\right)-x_{1}\left(t_{n}\right)}{\left(t_{n+1}-t_{n}\right)^{q_{1}}} \Gamma\left(1+q_{1}\right)=f_{1}\left(x_{1}(t), x_{2}(t), x_{3}(t), A_{1}\right), \\
& \frac{x_{2}\left(t_{n+1}\right)-x_{2}\left(t_{n}\right)}{\left(t_{n+1}-t_{n}\right)^{q_{2}}} \Gamma\left(1+q_{2}\right)=f_{2}\left(x_{1}(t), x_{2}(t), x_{3}(t), A_{2}\right), \\
& \frac{x_{3}\left(t_{n+1}\right)-x_{3}\left(t_{n}\right)}{\left(t_{n+1}-t_{n}\right)^{q_{3}}} \Gamma\left(1+q_{3}\right)=f_{3}\left(x_{1}(t), x_{2}(t), x_{3}(t), A_{3}\right) .
\end{aligned}
$$

Now we call model (15) as discrete fractional dynamic model.

In the rest of this paper, we will mainly study model (15). We assume that the triple $\left(x_{1}\left(t_{i}\right), x_{2}\left(t_{i}\right), x_{3}\left(t_{i}\right)\right), i=1,2, \ldots$, $N$, depicts the actual economic data. In order to make the model depict the relationship of the actual macroeconomic variables accurately at each time point, the estimation of $\widehat{B}_{i}=$ $\left(\widehat{q}_{i}, \widehat{A}_{i}\right)(i=1,2,3)$ is required to be subjected to the following condition:

$$
\begin{aligned}
& \left(\widehat{B}_{1}, \widehat{B}_{2}, \widehat{B}_{3}\right) \\
& =\arg \min \sum_{i=1}^{3} \sum_{k=1}^{N}\left(\frac{\Delta x_{i}\left(t_{k}\right)}{h^{q_{i}}} Q\left(q_{i}\right)\right. \\
& \left.\quad-f_{i}\left(x_{1}\left(t_{k}\right), x_{2}\left(t_{k}\right), x_{3}\left(t_{k}\right), A_{i}\right)\right)^{2},
\end{aligned}
$$

where $\Delta x_{i}\left(t_{k}\right)=x_{i}\left(t_{k+1}\right)-x_{i}\left(t_{k}\right), h=t_{k+1}-t_{k}$, and $Q\left(q_{i}\right)=$ $\Gamma\left(1+q_{i}\right)$.

It is easy to find that there are not common parameters for three equations of the model (14). Thus, the problem (16) is equivalent to three independent problems which are expressed as

$$
\begin{aligned}
& \left(\widehat{q}_{i}, \widehat{A}_{i}\right) \\
& =\arg \min \sum_{n=1}^{N-1}\left(\frac{\Delta x_{i}\left(t_{k}\right)}{h^{q_{i}}} Q\left(q_{i}\right)\right. \\
& \left.-f_{i}\left(x_{1}\left(t_{k}\right), x_{2}\left(t_{k}\right), x_{3}\left(t_{k}\right), A_{i}\right)\right)^{2} \\
& =\arg \min \frac{\Gamma^{\prime 2}\left(q_{i}\right)}{h^{2 q_{i}}} \sum_{n=1}^{N-1}\left(\Delta x_{i}\left(t_{k}\right)\right. \\
& \left.\quad-f_{i}\left(x_{1}\left(t_{k}\right), x_{2}\left(t_{k}\right), x_{3}\left(t_{k}\right), \widetilde{A}_{i}\right)\right)^{2},
\end{aligned}
$$

where

$$
\widetilde{A}_{i}=\frac{h^{q_{i}}}{\mathrm{Q}\left(q_{i}\right)} A_{i}, \quad i=1,2,3 .
$$

In (16), the minimum of the summation part is independent of the order $q_{i}, i=1,2,3$; thus the problem is equivalent to

$$
\begin{gathered}
\widehat{q}_{i}=\arg \min \left\{\Gamma\left(q_{i}+1\right)\left(t_{n+1}-t_{n}\right)^{q_{i}}\right\} \\
\widehat{A}_{i}^{o}=\arg \min \sum_{n=1}^{N-1}\left(\Delta x_{i}\left(t_{k}\right)-f_{i}\left(x_{1}(t), x_{2}(t), x_{3}(t), \widetilde{A}_{i}\right)\right)^{2}, \\
\widehat{A}_{i}=\frac{Q\left(q_{i}\right)}{h^{q_{i}}} \widehat{A}_{i}^{o}, \quad i=1,2,3 .
\end{gathered}
$$

We denote $X_{1 i}=x_{1}\left(t_{i}\right), X_{2 i}=x_{2}\left(t_{i}\right), X_{3 i}=x_{3}\left(t_{i}\right), X_{4 i}=$ $x_{1}\left(t_{i}\right) x_{2}\left(t_{i}\right), X_{5 i}=x_{2}\left(t_{i}\right) x_{3}\left(t_{i}\right), X_{6 i}=x_{1}\left(t_{i}\right) x_{3}\left(t_{i}\right), X_{7 i}=$ $x_{1}^{2}\left(t_{i}\right), X_{8 i}=x_{2}^{2}\left(t_{i}\right), X_{9 i}=x_{3}^{2}\left(t_{i}\right), i=1,2, \ldots, N-1$. Similar with the procedures of estimating the multivariate regression by the method of least squares, we can obtain the least squares estimation of (19) as

$$
\widehat{A}_{i}=\left(X^{T} X\right)^{-1} X^{T} Y_{i}, \quad i=1,2,3,
$$

where

$$
X=\left(\begin{array}{ccccc}
1 & X_{11} & X_{12} & \cdots & X_{19} \\
1 & X_{21} & X_{22} & \cdots & X_{29} \\
\vdots & \vdots & \vdots & \vdots & \vdots \\
1 & X_{(N-1) 1} & X_{(N-1) 2} & \cdots & X_{(N-1) 9}
\end{array}\right)
$$

and $Y_{i}=\left(x_{i}\left(t_{2}-t_{1}\right), x_{i}\left(t_{3}-t_{2}\right), \ldots, x_{i}\left(t_{N}-t_{N-1}\right)\right)^{T}, i=1,2,3$. The superscript $T$ indicates the transposition of matrix and vector.

Therefore, we obtain the estimation of $\left(\widehat{q}_{i}, \widehat{A}_{i}\right), i=1,2,3$, as

$$
\begin{gathered}
\widehat{A}_{i}=\frac{\Gamma\left(\widehat{q}_{i}+1\right)}{t_{n+1}-t_{n}}\left(X^{T} X\right)^{-1} X^{T} Y_{i}, \\
\widehat{q}_{i}=\arg \min \left\{\Gamma\left(q_{i}+1\right)\left(t_{n+1}-t_{n}\right)^{1-q_{i}}\right\} .
\end{gathered}
$$

Remark 1. It is easy to find that the estimation of $\widehat{q}_{i}, i=1,2,3$, is not related to the sample observations; then $\widehat{q}_{1}=\widehat{q}_{2}=\widehat{q}_{3}$.

Remark 2. The estimation for the $\left(\widehat{q}_{i}, \widehat{A}_{i}\right)(i=1,2,3)$ in the model (10) with Jumarie's definition of fractional derivative is unique.

\section{Application of Discrete Fractional Order Economic System Model: Evidence from US}

In this section, we present an application of discrete fractional order economic model based on the macroeconomic data of USA, which demonstrates the effectiveness of model in simulating the evolution of the macroeconomic variables and predicting the future behavior of the macroeconomic system.

4.1. Data Description. In fractional economic model (12), the GDP, inflation, and unemployment rate are governed by a 


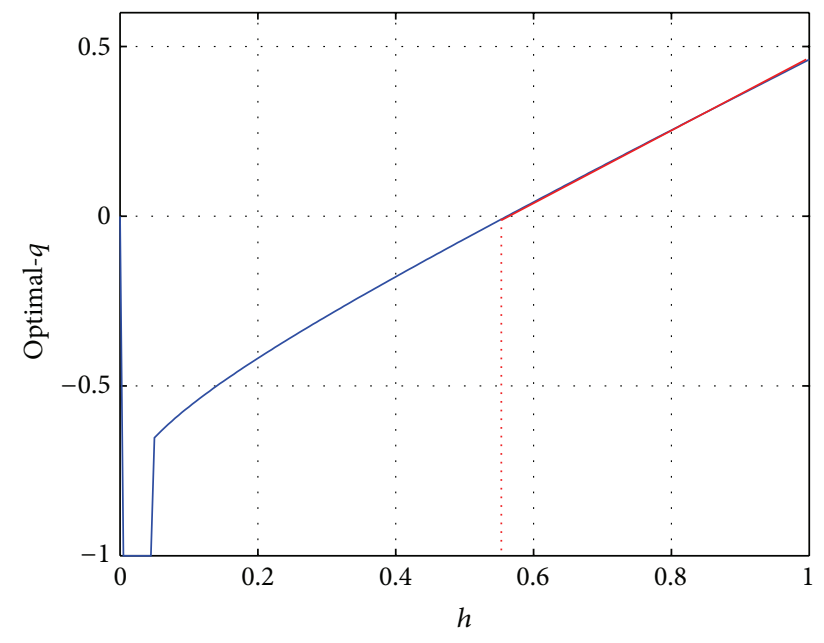

FIGURE 1: The optimal estimation of fractional order $q$ in (12).

nonlinear fractional dynamical system. The percent change of GDP, average consumer prices percent change rate, unemployment rate percent of total labor force are used to reflect the variables GDP, inflation, and unemployment, respectively. The annual data starts from year 1980 to 2011. The resource of data about percent change of GDP, average consumer prices percent change rate, and unemployment rate percent of total labor force is EconStats which is organized by IMF.

4.2. The Estimation of Parameters in the Economic Models. We first estimate the optimal fractional order. In (25), the optimal value of the order is influenced by the time step size of discretization; that is, $h=t_{n+1}-t_{n}$. We compute the optimal order with different time step sizes. The relation between the optimal value of $q$ and time step $h$ is shown in Figure 1. In this figure, we observe that as a parameter, the step size $h$ in discrete economic system cannot be too small. From the study on the macroeconomic data of USA, when $h \leq 0.55$, the fractional order turns out to be negative, which contradicts the basic definition of fractional derivative (see (9)). It is also illustrated in Figure 1 that we have lots of choices for step size $h$, marked by red color, when discretizing the original continuous model (14).

Now we estimate the left parameters. To make a better comparison of numerical simulation, we fit the parameters of fractional economic model (12) with different time step sizes as $h=0.65,0.75$, and 0.85 . Their corresponding estimations of optimal fractional order are 0.0951, 0.2011, and 0.3059 , which are also easily checked in Figure 1 . The details of empirical results of the economic system (12) are shown in Tables 1, 2, and 3.

In Table 1 , the estimation results of parameters in the macroeconomic models (1) and (12) are shown with $h=0.65$, respectively. From this table, it is known that the sum squared residuals (SSR) of every equation are less than 0.01 , which demonstrates that the estimation result is reasonable. Moreover, the SSR value in the nonlinear model (12) is less than that of the corresponding equation in the linear model (1), which shows that the nonlinear discrete fractional economic model
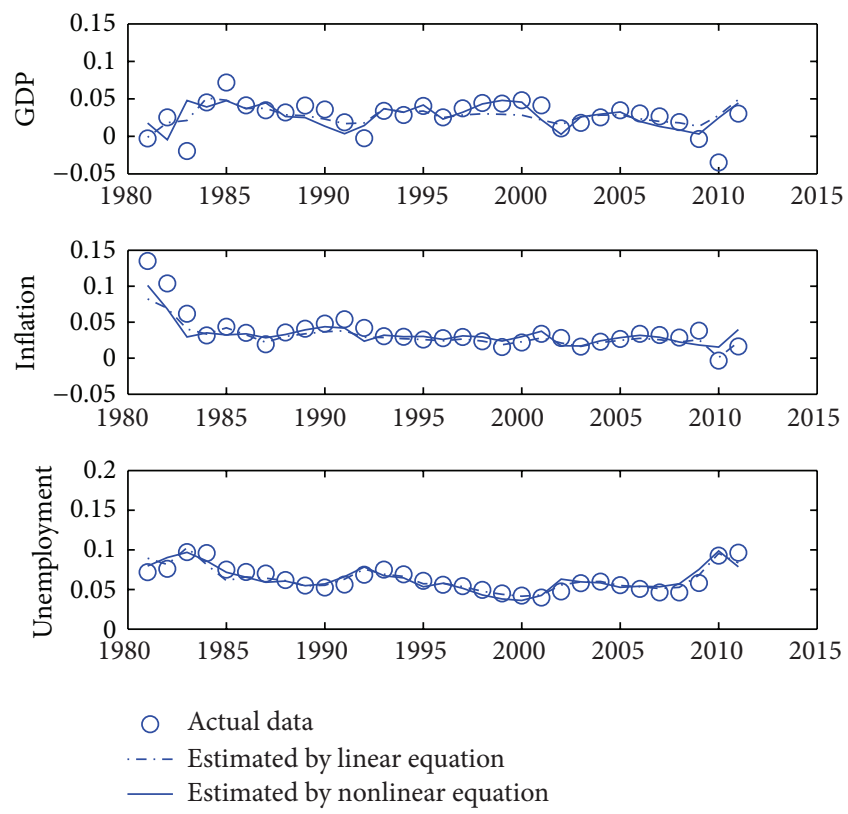

FIGURE 2: The actual and estimated data of the GDP, inflation, and unemployment by the linear and nonlinear models with $h=0.65$.

is more accurate than that of linear case. Furthermore, we observe that the $R$-squared values of nonlinear case are larger than 0.6 , while the $R$-squared values of linear case are smaller than 0.6 , which also demonstrates that the nonlinear model (12) is better than linear model (1). In order to illustrate the effectiveness of the discrete model (12) intuitively, the actual data and the data generated by the linear model (1) and the nonlinear model (12) with step size $h=0.65$ are plotted in Figure 2. From this figure, it can be easily found that the nonlinear model (12) depicts the actual data more accurately than the linear model (1).

Table 2 shows the estimation results of the parameters in linear model (1) and nonlinear model (12) with step size $h=0.75$, respectively. From Table 2, we observe that the SSR value is smaller than 0.01 , which demonstrates that the estimation is reasonable. Moreover, the SSR value of nonlinear model (12) is smaller than that of linear model (1), which shows that the nonlinear model (12) is much better than the linear model (1) for describing the USA macroeconomic data. Furthermore, the $R$-squared value of each equation in nonlinear model (12) is greater than that in the linear model (1), which demonstrates that the nonlinear model (12) is more accurate than the linear model (1). To illustrate the observation more clearly, the actual data and data estimated by the linear and nonlinear models with step size $h=0.75$ are shown in Figure 3. From this plot, we easily find that the data governed by the nonlinear model (12) fit the actual data curve much better.

In Table 3, the estimation results of the parameters in linear model (1) and nonlinear model (12) with step size $h=0.85$ are given, respectively. Again, similar with two previous tables, we observe that the SSR value of each equation in the nonlinear model (12) is less than that in the linear model (1), which once again demonstrates that the nonlinear model 
TABLE 1: The estimation results of parameters in model (12) with $h=0.65$.

\begin{tabular}{|c|c|c|c|c|c|c|c|c|}
\hline \multirow{2}{*}{$A_{1}$} & \multicolumn{2}{|c|}{$D_{t}^{q_{1}} x_{1}(t)$} & \multirow{2}{*}{$A_{2}$} & \multicolumn{2}{|c|}{$D_{t}^{q_{2}} x_{2}(t)$} & \multirow{2}{*}{$A_{3}$} & \multicolumn{2}{|c|}{$D_{t}^{q_{3}} x_{3}(t)$} \\
\hline & $\mathrm{L}$ & NL & & $\mathrm{L}$ & NL & & $\mathrm{L}$ & NL \\
\hline$c_{1}$ & -0.0050 & -0.0753 & $c_{2}$ & 0.0016 & -0.0407 & $c_{3}$ & 0.0215 & 0.0754 \\
\hline$a_{11}$ & -0.6284 & 1.0035 & $a_{21}$ & 0.1551 & 1.2353 & $a_{31}$ & -0.2587 & -1.4196 \\
\hline$a_{12}$ & -0.2203 & -0.6022 & $a_{22}$ & -0.4282 & 0.5073 & $a_{32}$ & 0.1220 & -0.1173 \\
\hline$a_{13}$ & 0.4818 & 2.1463 & $a_{23}$ & 0.1034 & 0.4666 & $a_{33}$ & -0.2939 & -1.3398 \\
\hline$a_{14}$ & & -16.778 & $a_{24}$ & & -2.7541 & $a_{34}$ & & 7.3886 \\
\hline$a_{15}$ & & 4.7152 & $a_{25}$ & & -18.606 & $a_{35}$ & & 4.4934 \\
\hline$a_{16}$ & & -16.371 & $a_{26}$ & & -9.1467 & $a_{36}$ & & 10.295 \\
\hline$a_{17}$ & & 4.7435 & $a_{27}$ & & -4.9194 & $a_{37}$ & & 2.8206 \\
\hline$a_{18}$ & & 1.2345 & $a_{28}$ & & 3.6713 & $a_{38}$ & & -1.0608 \\
\hline$a_{19}$ & & -10.108 & $a_{29}$ & & 3.6943 & $a_{39}$ & & 4.0766 \\
\hline SSR & $9.3 e-3$ & $5.9 e-3$ & SSR & $3.2 e-3$ & $1.9 e-3$ & SSR & $1.6 e-3$ & $9.3 e-4$ \\
\hline$R^{2}$ & 0.4955 & 0.6803 & $R^{2}$ & 0.5615 & 0.7399 & $R^{2}$ & 0.4705 & 0.6918 \\
\hline
\end{tabular}

Note: SSR is the sum squared residuals. L stands for the linear economic model (1). NL stands for the nonlinear economic model (14). $R$ stands for the $R$-squared value.

TABLE 2: The estimation results of parameters in model (12) with $h=0.75$.

\begin{tabular}{|c|c|c|c|c|c|c|c|c|}
\hline \multirow{2}{*}{$A_{1}$} & \multicolumn{2}{|c|}{$D_{t}^{q_{1}} x_{1}(t)$} & \multirow{2}{*}{$A_{2}$} & \multicolumn{2}{|c|}{$D_{t}^{q_{2}} x_{2}(t)$} & \multirow{2}{*}{$A_{3}$} & \multicolumn{2}{|c|}{$D_{t}^{q_{3}} x_{3}(t)$} \\
\hline & $\mathrm{L}$ & $\mathrm{NL}$ & & $\mathrm{L}$ & NL & & $\mathrm{L}$ & NL \\
\hline$c_{1}$ & -0.0049 & -0.0737 & $c_{2}$ & 0.0016 & -0.0398 & $c_{3}$ & 0.0211 & 0.0739 \\
\hline$a_{11}$ & -0.6154 & 0.9826 & $a_{21}$ & 0.1519 & 1.2096 & $a_{31}$ & -0.2533 & -1.3900 \\
\hline$a_{12}$ & -0.2157 & -0.5897 & $a_{22}$ & -0.4193 & 0.4967 & $a_{32}$ & 0.1194 & -0.1148 \\
\hline$a_{13}$ & 0.4718 & 2.1016 & $a_{23}$ & 0.1013 & 0.4569 & $a_{33}$ & -0.2878 & -1.3118 \\
\hline$a_{14}$ & & -16.428 & $a_{24}$ & & -2.6967 & $a_{34}$ & & 7.2346 \\
\hline$a_{15}$ & & 4.6169 & $a_{25}$ & & -18.219 & $a_{35}$ & & 4.3997 \\
\hline$a_{16}$ & & -16.030 & $a_{26}$ & & -8.9561 & $a_{36}$ & & 10.080 \\
\hline$a_{17}$ & & 4.6446 & $a_{27}$ & & -4.8169 & $a_{37}$ & & 2.7619 \\
\hline$a_{18}$ & & 1.2087 & $a_{28}$ & & 3.5948 & $a_{38}$ & & -1.0387 \\
\hline$a_{19}$ & & -9.8971 & $a_{29}$ & & 3.6173 & $a_{39}$ & & 3.9917 \\
\hline SSR & $8.9 e-3$ & $5.6 e-3$ & SSR & $3.1 e-3$ & $1.8 e-3$ & SSR & $1.5 e-3$ & $8.9 e-4$ \\
\hline$R^{2}$ & 0.4955 & 0.6803 & $R^{2}$ & 0.5615 & 0.7399 & $R^{2}$ & 0.4705 & 0.6918 \\
\hline
\end{tabular}

TABLE 3: The estimation results of parameters in model (12) with $h=0.85$.

\begin{tabular}{|c|c|c|c|c|c|c|c|c|}
\hline \multirow{2}{*}{$A_{1}$} & \multicolumn{2}{|c|}{$D_{t}^{q_{1}} x_{1}(t)$} & \multirow{2}{*}{$A_{2}$} & \multicolumn{2}{|c|}{$D_{t}^{q_{2}} x_{2}(t)$} & \multirow{2}{*}{$A_{3}$} & \multicolumn{2}{|c|}{$D_{t}^{q_{3}} x_{3}(t)$} \\
\hline & $\mathrm{L}$ & NL & & $\mathrm{L}$ & NL & & $\mathrm{L}$ & NL \\
\hline$c_{1}$ & -0.0048 & -0.0714 & $c_{2}$ & 0.0016 & -0.0386 & $c_{3}$ & 0.0204 & 0.0716 \\
\hline$a_{11}$ & -0.5962 & 0.9521 & $a_{21}$ & 0.1472 & 1.1721 & $a_{31}$ & -0.2454 & -1.3468 \\
\hline$a_{12}$ & -0.2090 & -0.5714 & $a_{22}$ & -0.4063 & 0.4813 & $a_{32}$ & 0.1157 & -0.1113 \\
\hline$a_{13}$ & 0.4572 & 2.0364 & $a_{23}$ & 0.0981 & 0.4427 & $a_{33}$ & -0.2789 & -1.2711 \\
\hline$a_{14}$ & & -15.918 & $a_{24}$ & & -2.6130 & $a_{34}$ & & 7.0099 \\
\hline$a_{15}$ & & 4.4736 & $a_{25}$ & & -17.653 & $a_{35}$ & & 4.2631 \\
\hline$a_{16}$ & & -15.532 & $a_{26}$ & & -8.6779 & $a_{36}$ & & 9.7671 \\
\hline$a_{17}$ & & 4.5004 & $a_{27}$ & & -4.6673 & $a_{37}$ & & 2.6761 \\
\hline$a_{18}$ & & 1.1712 & $a_{28}$ & & 3.4831 & $a_{38}$ & & -1.0065 \\
\hline$a_{19}$ & & -9.5897 & $a_{29}$ & & 3.5050 & $a_{39}$ & & 3.8677 \\
\hline SSR & $8.3 e-3$ & $5.3 e-3$ & SSR & $2.9 e-3$ & $1.7 e-3$ & SSR & $1.4 e-3$ & $8.3 e-4$ \\
\hline$R^{2}$ & 0.4955 & 0.6803 & $R^{2}$ & 0.5615 & 0.7399 & $R^{2}$ & 0.4705 & 0.6918 \\
\hline
\end{tabular}



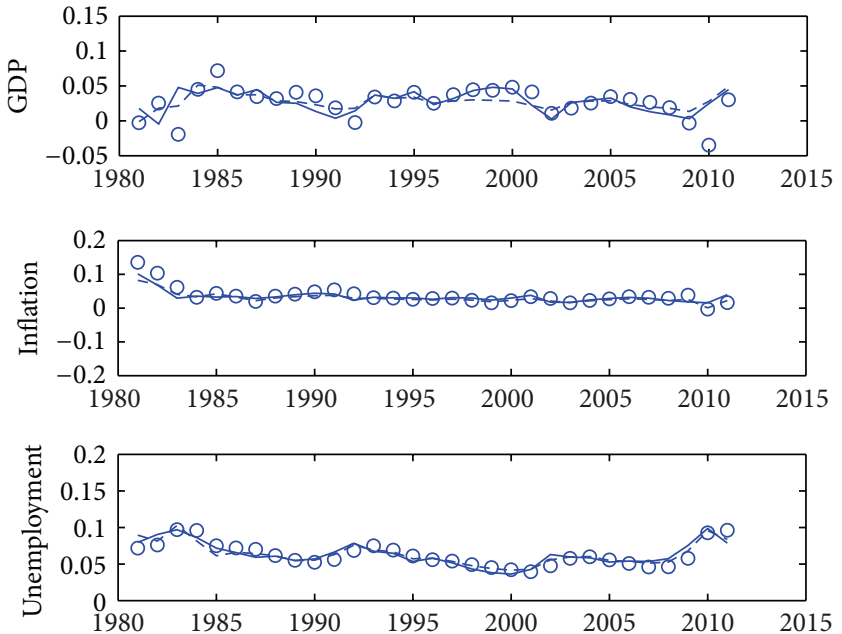

- Actual data

- - - Estimated by linear equation

_ Estimated by nonlinear equation

FIGURE 3: The actual and estimated data of the GDP, inflation, and unemployment by the linear and nonlinear models with $h=0.75$.

(12) is more effective than the linear model (1). Furthermore, we also notice that the $R$-squared value of each equation in nonlinear model (12) is greater than that in the linear model (1), which demonstrates that the nonlinear model (12) is more accurate than the linear model (1). To illustrate the analysis more clearly, we draw relation of the actual data and the data estimated by the linear and nonlinear models with step size $h=0.85$ in Figure 4. From this figure, we find that the nonlinear model (12) is much better in describing the macroeconomic data.

To end this subsection, we would like to make the following remark.

Remark 3. The discrete nonlinear fractional model (12) considered in our paper is better than the linear economic model in [22], because of the fact that the nonlinear terms involved in our model can describe the relation of macroeconomic variables better. Taking the example of the macroeconomic data of USA, we find that as the step size approaches one, the discrete economic model (12) that we obtained becomes more accurate.

4.3. Further Discussion of the Nonlinear Model (12). Now we simulate the future behavior of the macroeconomic variables considered in the nonlinear model (12). In fact, (15) provides a recursive formula as

$$
\begin{aligned}
& x_{1}\left(t_{n}+1\right) \\
& \quad=x_{1}\left(t_{n}\right)+\frac{h^{\widehat{q}_{1}}}{\Gamma\left(1+\widehat{q}_{1}\right)} f_{1}\left(x_{1}(t), x_{2}(t), x_{3}(t), A_{1}\right),
\end{aligned}
$$
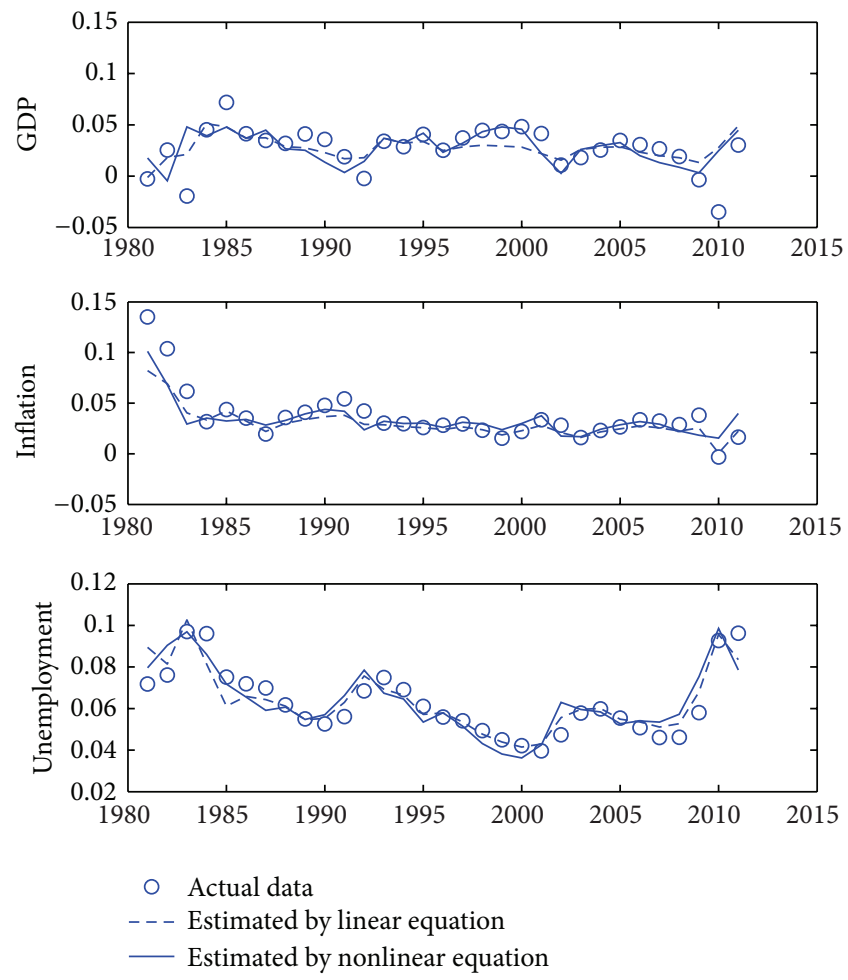

FIGURE 4: The actual and estimated data of the GDP, inflation and unemployment by the linear and nonlinear models with $h=0.85$.

$$
\begin{aligned}
& x_{2}\left(t_{n}+1\right) \\
& \quad=x_{2}\left(t_{n}\right)+\frac{h^{\widehat{q}_{2}}}{\Gamma\left(1+\hat{q}_{2}\right)} f_{2}\left(x_{1}(t), x_{2}(t), x_{3}(t), A_{2}\right), \\
& x_{3}\left(t_{n}+1\right) \\
& \quad=x_{3}\left(t_{n}\right)+\frac{h^{\widehat{q}_{3}}}{\Gamma\left(1+\widehat{q}_{i}\right)} f_{3}\left(x_{1}(t), x_{2}(t), x_{3}(t), A_{3}\right),
\end{aligned}
$$

where $n=1,2, \ldots$

The in-of-sample prediction can be calculated by the above recursive equations. The actual data of GDP, inflation, and unemployment rate are compared with their corresponding in-of-sample prediction with step size $h=0.85$. The results with the linear and nonlinear models are shown in Figures 5 and 6, respectively.

In Figure 5, the comparison of actual data and $N$-step predicting (the procedure of the $T$-step predicting method is as follows: suppose that we have a time series $a=\left\{a_{i}\right\}_{i=0,1,2, \ldots}$ that may be governed by a discrete system $a_{k+1}=g\left(a_{k}\right), k=$ $0,1,2, \ldots$. Now for checking how accurate the function $g(\cdot)$ is, we can construct a new time series $\bar{a}=\left\{\bar{a}_{i}\right\}, i=0,1,2, \ldots$, where $\bar{a}_{j}=\stackrel{T \text { times }}{\mathfrak{g} \cdot \mathrm{g}}\left(a_{j-T}\right), j \geq T$, and $\bar{a}_{j}=a_{j}$, when $0 \leq j<T$. Then the more close to zero the $\|\bar{a}-a\|_{2}$ is, the better the function $g$ is) data ( $T=2,3,4)$ of the GDP, inflation, and unemployment with linear model (1) are illustrated separately from top to the bottom. In each subfigure, we observe that the 

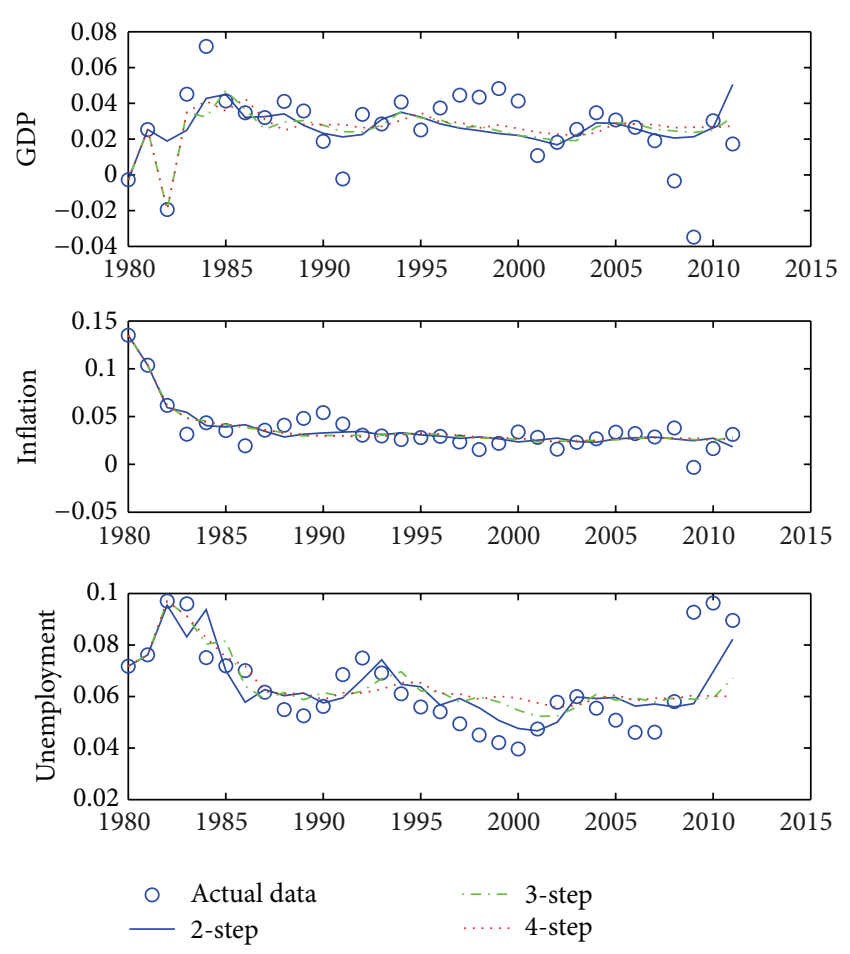

FIGURE 5: The actual data and in-of-sample prediction of the GDP, inflation, and unemployment with the linear model (1).

2-step predicting data fit the actual data better than the other results of two cases. Specially, in the third subfigure, we notice that the 2-step predicting data line (marked by solid blue) goes through the critical points, which demonstrates that the result by 2-step predicting method is better than 3- and 4-step predicting data in the linear model (1).

In Figure 6, the in-of-sample predictions of the macroeconomic variables of nonlinear dynamic model (12) are shown individually in three subfigures, respectively. The result of GDP is shown in the first subfigure by using the 2-, 3-, and 4-step predicting method. We observe that in this case, the result obtained by 4 -step predicting method is better than the left two cases. This is different from the conclusion shown in Figure 5 because here we consider the nonlinear macroeconomic model, which has been verified to be better. In the other two subfigures, we also find that the lines of 2-step predicting data are more accurate than the others.

Furthermore, careful comparison between the Figures 5 and 6 shows that the lines of 2-step predicting data in Figure 6 describe the actual data better than that in Figure 5, which again demonstrates the excellence of the nonlinear model (12). In Figures 5 and 6, we only present results of $T=2,3,4$. However, one can further examine $T \geq 5$ and similar analysis and conclusions can be drawn.

\section{Conclusions}

In this work, we construct a new dynamic nonlinear economic model with fractional derivative defined by Jumarie's sense. The corresponding discrete model is drawn by removing the limit operator in Jumarie's fractional derivative.
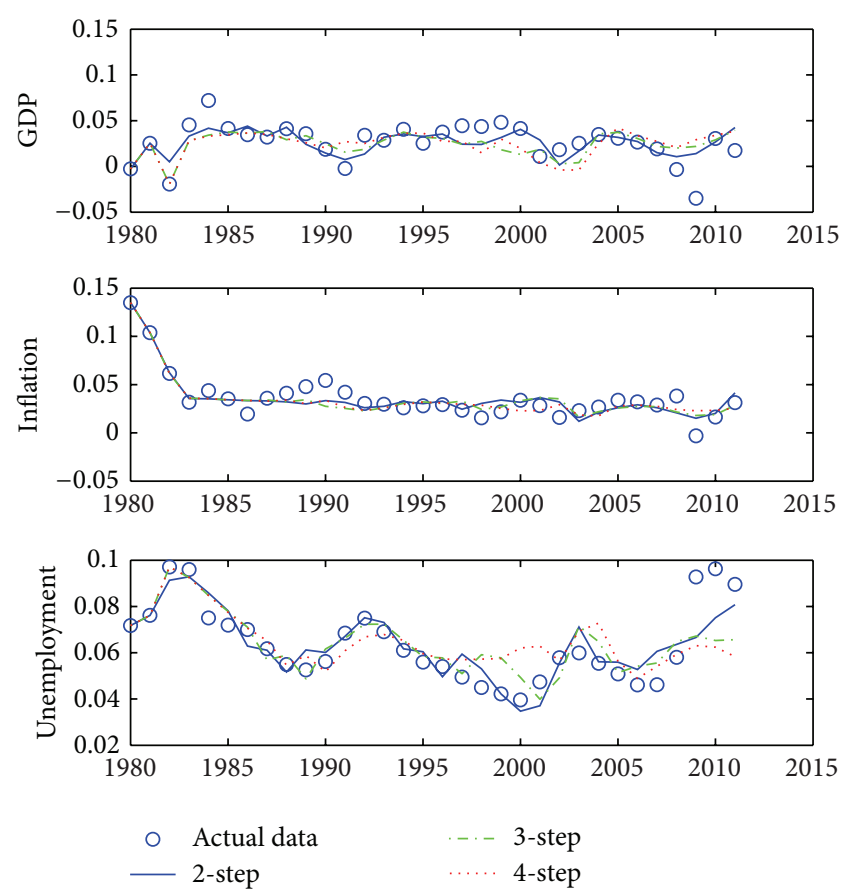

FIGURE 6: The actual data and in-of-sample prediction of the GDP, inflation, and unemployment with the nonlinear model (12).

The step size in discretization and the fractional order are regarded as parameters in the obtained discrete model. All the parameters are estimated by the least square method. Based on the macroeconomic data, we calculate the optimal fractional order and step size. We verify that there are lots of choices of values of step size in discretization and every step size has a unique optimal fractional order. The sum squared residuals and the $R$-squared values of estimation in simulation are computed, which demonstrate that the nonlinear discrete model is effective in modelling the macroeconomic variables of USA, and it is better than the linear model proposed in the references $[22,24]$.

Although we only consider the macroeconomic variables, that is, GDP, inflation, and unemployment of USA, many other inner-connected macroeconomic variables can be considered using the same method. Our modelling methodology can also be applied to other nations to investigate their evolution of economic variables.

\section{Acknowledgments}

The authors sincerely thank the reviewers for their constructive comments which significantly improved the quality of their paper. This work is partly supported by the Philosophy and Social Science Fund Project (no. 11YBA097) and the Scientific Research Funding of Hunan Provincial Education Department (no. 11C0437). The authors would also like to express sincere appreciation to Dr. Lei He and Dr. Shichang Ma for their unselfish help and benefit discussion on the preparation of the paper. 


\section{References}

[1] R. Goodwin, Chaotic Economic Dynamics, Oxford University Press, New York, NY, USA, 1990.

[2] R. Shone, Economic Dynamics, Cambridge University Press, New York, NY, USA, 2nd edition, 2002.

[3] J. Stachurski, Economic Dynamics: Theory and Computation, MIT Press, Cambridge, Mass, USA, 2009.

[4] A. C.-L. Chian, F. A. Borotto, E. L. Rempel, and C. Rogers, "Attractor merging crisis in chaotic business cycles," Chaos, Solitons \& Fractals, vol. 24, no. 3, pp. 869-875, 2005.

[5] J. H. Ma and Y. S. Chen, "Study for the bifurcation topological structure and the global complicated character of a kind of nonlinear finance system. I," Applied Mathematics and Mechanics, vol. 22, no. 11, pp. 1240-1251, 2001.

[6] J. H. Ma and Y. S. Chen, "Study for the bifurcation topological structure and the global complicated character of a kind of nonlinear finance system. II," Applied Mathematics and Mechanics, vol. 22, no. 12, pp. 1375-1382, 2001.

[7] I. Podlubny, Fractional Differential Equations, vol. 198 of Mathematics in Science and Engineering, Academic Press, New York, NY, USA, 1999.

[8] A. A. Kilbas, H. M. Srivastava, and J. J. Trujillo, Theory and Applications of Fractional Differential Equations, vol. 204 of NorthHolland Mathematics Studies, Elsevier, Amsterdam, The Netherlands, 2006.

[9] Y. Xu and Z. He, "The short memory principle for solving Abel differential equation of fractional order," Computers \& Mathematics with Applications, vol. 62, no. 12, pp. 4796-4805, 2011.

[10] Y. Xu and Z. He, "Synchronization of variable-order fractional financialsystem via active control method," Central European Journal of Physics, vol. 11, no. 6, pp. 824-835, 2013.

[11] Y. Xu and Z. He, "Existence and uniqueness results for Cauchy problem of variable-order fractional differential equations," Journal of Applied Mathematics and Computing, vol. 43, no. 12, pp. 295-306, 2013.

[12] Y. Xu, Z. He, and Q. Xu, "Numerical solutions of fractional advectiondiffusion equations with a kind of new generalized fractional derivative," International Journal of Computer Mathematics, 2013.

[13] Y. Xu and O. P. Agrawal, "Models and numerical schemes for generalized van der Pol equations," Communications in Nonlinear Science and Numerical Simulation, vol. 18, no. 12, pp. 35753589, 2013.

[14] S. Ma, Y. Xu, and W. Yue, "Numerical solutions of a variableorder fractional financial system," Journal of Applied Mathematics, vol. 2012, Article ID 417942, 14 pages, 2012.

[15] A. Kirman and G. Teyssiere, "Microeconomic models for long memory in the volatility of financial time series," Studies in Nonlinear Dynamics and Econometrics, vol. 5, no. 4, pp. 281-302, 2002.

[16] K. Yamasaki, L. Muchnik, S. Havlin, A. Bunde, and H. E. Stanley, "Scaling and memory in volatility return intervals in financial markets," Proceedings of the National Academy of Sciences of the United States of America, vol. 102, no. 26, pp. 9424-9428, 2005.

[17] V. Anh and A. Inoue, "Financial markets with memory. I. Dynamic models," Stochastic Analysis and Applications, vol. 23, no. 2, pp. 275-300, 2005.

[18] F. Garzarelli, M. Cristelli, A. Zaccaria, and L. Pietronero, "Memory effects in stock price dynamics: evidences oftechnical trading," http://arxiv.org/abs/1110.5197.
[19] W.-C. Chen, "Nonlinear dynamics and chaos in a fractionalorder financial system," Chaos, Solitons \& Fractals, vol. 36, no. 5, pp. 1305-1314, 2008.

[20] S. Dadras and H. R. Momeni, "Control of a fractional-order economical system via sliding mode," Physica A, vol. 389, no. 12, pp. 2434-2442, 2010.

[21] Z. Wang, X. Huang, and G. Shi, "Analysis of nonlinear dynamics and chaos in a fractional order financial system with time delay," Computers \& Mathematics with Applications, vol. 62, no. 3, pp. 1531-1539, 2011.

[22] T. Skovranek and I. Podlubny, "Modeling of the national economics in state-space: a fractional calculus approach," Economic Modelling, vol. 29, no. 4, pp. 1322-1327, 2012.

[23] G. Jumarie, "Modified Riemann-Liouville derivative and fractional Taylor series of nondifferentiable functions further results," Computers \& Mathematics with Applications, vol. 51, no. 9-10, pp. 1367-1376, 2006.

[24] D. Gale, The Theory of Linear Economic Models, The University of Chicago Press, Chicago, Ill, USA, 1989. 


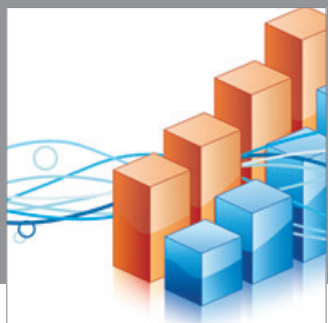

Advances in

Operations Research

mansans

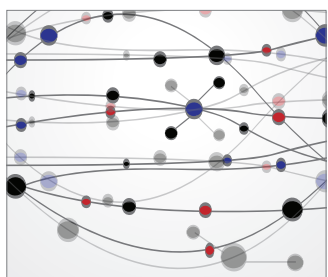

The Scientific World Journal
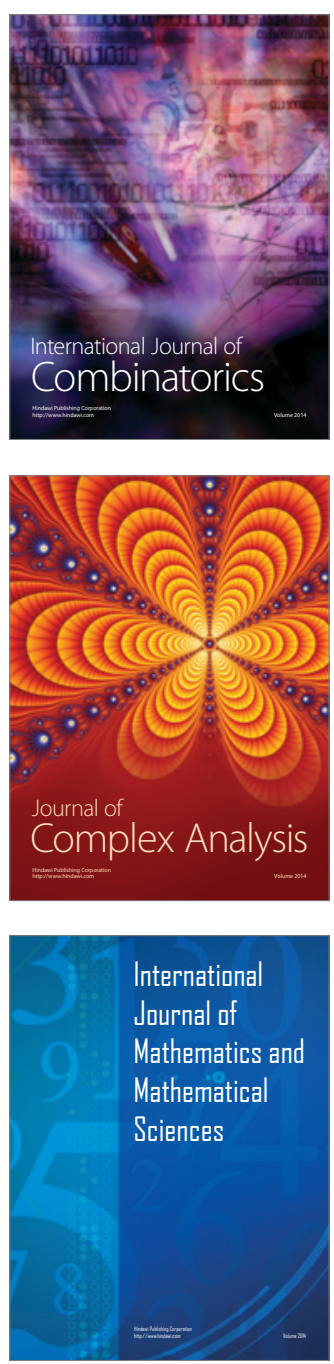
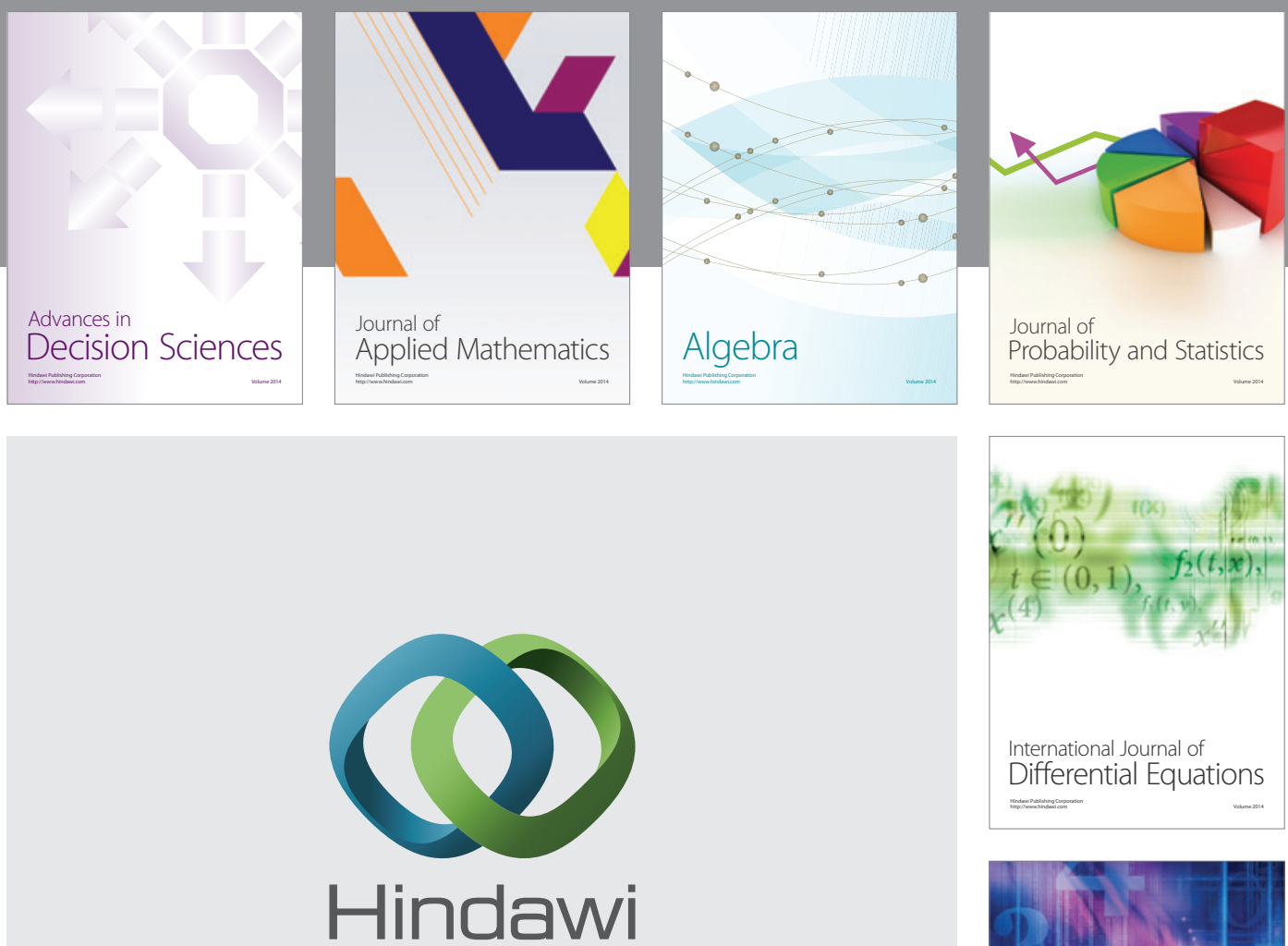

Submit your manuscripts at http://www.hindawi.com
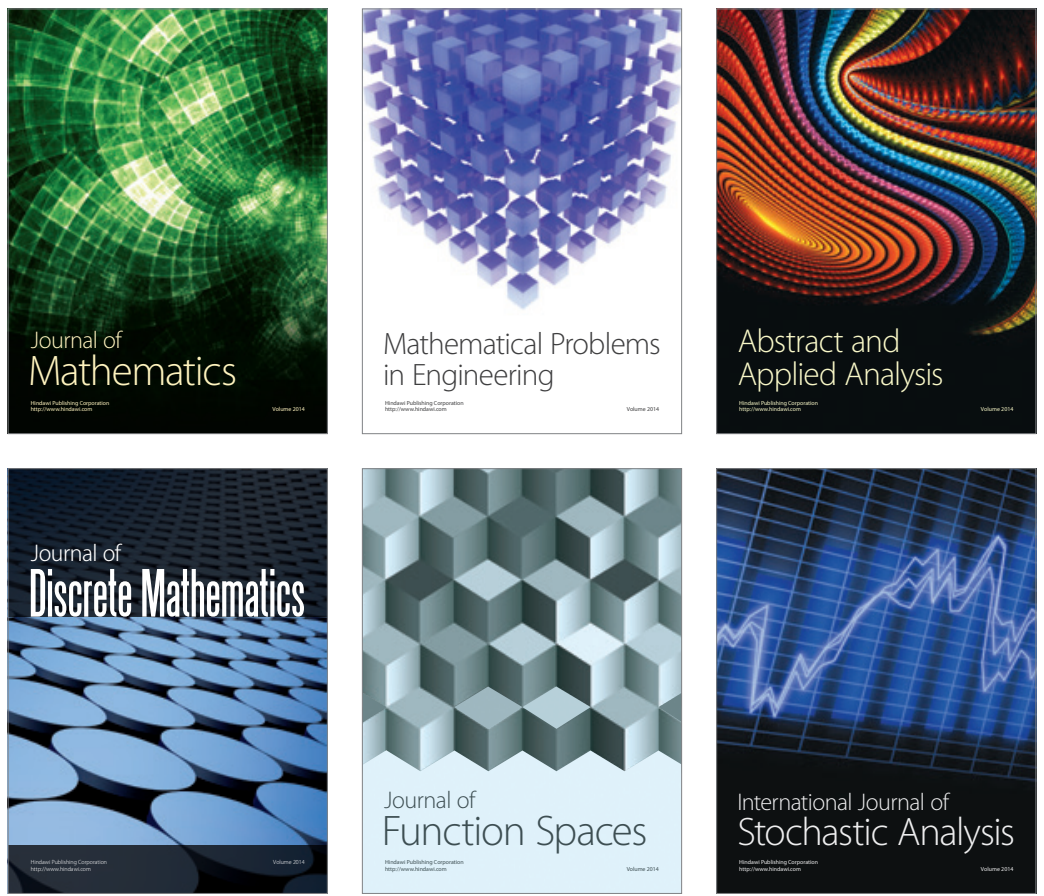

Journal of

Function Spaces

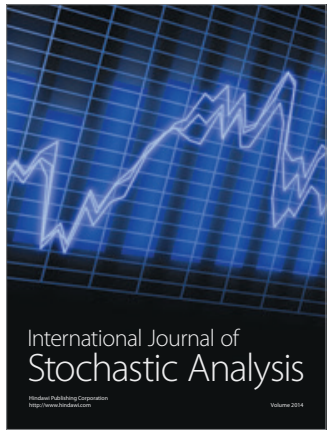

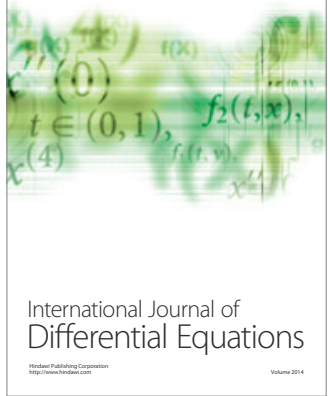
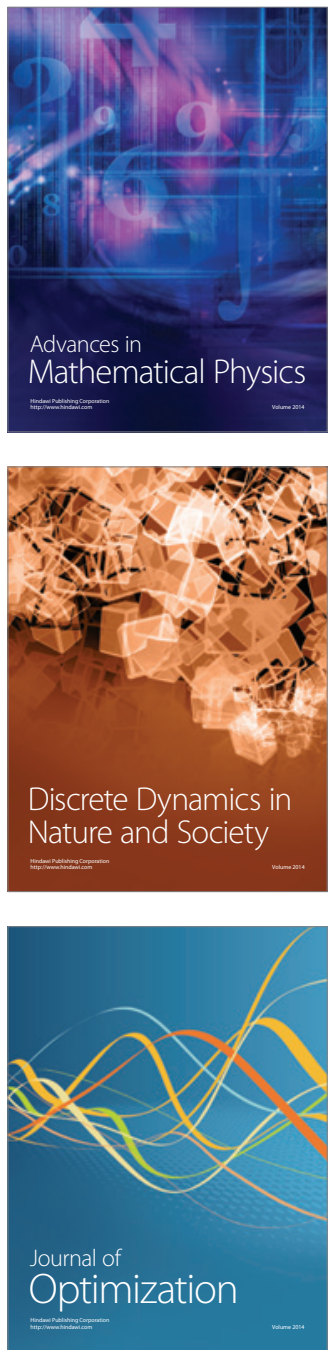